\title{
ARTICLE OPEN Restructuring of Enterococcus faecalis biofilm architecture in response to antibiotic-induced stress
}

\author{
Jennifer L. Dale ${ }^{1}$, Jennifer L. Nilson ${ }^{1}$, Aaron M. T. Barnes (iD ${ }^{1,2}$ and Gary M. Dunny ${ }^{1}$
}

Bacterial biofilms are intrinsically resistant to antimicrobial treatment, which contributes to microbial persistence in clinical infections. Enterococcus faecalis is an opportunistic pathogen that readily forms biofilms and is the most prevalent enterococcal species identified in healthcare-associated infections. Since intrinsic resistance to multiple antibiotics is common for enterococci, and antibiotic resistance is elevated in biofilm populations, it is imperative to understand the mechanisms involved. Previously, we identified two glycosyltransferase genes whose disruption resulted in impaired nascent biofilm formation in the presence of antibiotic concentrations subinhibitory for parent growth and biofilm formation. The glycosyltransferases are involved in synthesis of the cell-wall-associated rhamnopolysaccharide Epa. Here we examined the effect of epa mutations on the temporal development of $E$. faecalis biofilms, and on the effects of antibiotics on pre-formed biofilms using scanning electron microscopy. We show that $\triangle$ epaOX mutant cells arrange into complex multidimensional biofilms independent of antibiotic exposure, while parent cells form biofilms that are monolayers in the absence of antibiotics. Remarkably, upon exposure to antibiotics parent biofilm cells restructure into complex three-dimensional biofilms resembling those of the $\triangle$ epaOX mutant without antibiotics. All biofilms exhibiting complex cellular architectures were less structurally stable than monolayer biofilms, with the biofilm cells exhibiting increased detachment. Our results indicate that $E$. faecalis biofilms restructure in response to cellular stress whether induced by antibiotics in the case of parent cells, or by deficiencies in Epa composition for the $\triangle e p a O X$ strain. The data demonstrate a link between cellular architecture and antibiotic resistance of $E$. faecalis biofilms.

npj Biofilms and Microbiomes (2017)3:15; doi:10.1038/s41522-017-0023-4

\section{INTRODUCTION}

Antibiotic resistance is a significant burden in healthcareassociated infections (HAls), ${ }^{1}$ and is exacerbated by the presence of biofilms. It is estimated that biofilm populations are 10-1000 times more resistant to antibiotics than planktonic cells. ${ }^{2,3}$ Several diverse mechanisms have been implicated in enhanced biofilm resistance to antibiotics including decreased antibiotic penetration, sequestration of antibiotics, and the presence of persister cells. ${ }^{4-6}$

Enterococci are among the most common pathogens found in HAls with Enterococcus faecalis being the primary enterococcal species identified. ${ }^{1}$ E. faecalis readily forms biofilms on a widerange of natural and artificial substrates including indwelling medical devices, damaged heart valves, and catheters, which contributes to its pathogenicity. ${ }^{7,8}$ We identified recently two glycosyltransferase (GTF) genes that contribute to $E$. faecalis biofilm-associated antibiotic resistance. Deletion of either GTF epal or epaOX impaired nascent biofilm formation in the presence of subinhibitory concentrations of antibiotics, with negligible reduction of biofilm formation in the absence of antibiotics. ${ }^{9}$ The GTFs are involved in the synthesis of a cell-wall-associated polysaccharide and are linked to the enterococcal polysaccharide antigen (epa) gene cluster.

Various functions have been described for epa genes in E. faecalis. Certain epa genes are important determinants of Epa polysaccharide composition, biofilm formation, phage susceptibility, and cellular morphology. ${ }^{9-12}$ Deletion of epa genes resulted in decreased virulence in murine peritonitis and ascending urinary tract infection models, ${ }^{13,} 14$ impaired infectivity in Galleria mellonella, ${ }^{15}$ and diminished murine intestinal colonization. ${ }^{10}$ Our lab and others have shown that deletion of various epa genes decreased cell-wall integrity and increased cell envelope permeability. ${ }^{9,}{ }^{16}$ Recently, Hoff et al. ${ }^{16}$ demonstrated that defects in Epa polysaccharide production increased susceptibility of $E$. faecalis planktonic cells toward cephalosporin antibiotics. While the phenotypical consequences of Epa alterations continue to expand, our understanding of Epa structure and how it contributes to virulence and intrinsic antibiotic resistance remains incomplete.

In this study, we sought to determine how epal and epaOX enhance antibiotic resistance in $E$. faecalis during biofilm growth. Here we show that deletion of epaOX results in the most noticeable phenotypic differences in biofilm architecture and biochemical composition. We also provide evidence that exposure of parent biofilms to subinhibitory concentrations of antibiotics results in restructuring of biofilms into a complex threedimensional (3D) architecture reminiscent of $\triangle e p a O X$ biofilms grown in the absence of antibiotics. In addition, the structural stability of $E$. faecalis biofilms exhibiting increased 3D cellular architecture is significantly diminished relative to monolayer biofilms, even though biofilm biomass is not reduced. We propose that formation of a complex 3D biofilm structure in the presence of antibiotics, or as a result of cell-wall alterations, may constitute a stress response of enterococcal biofilm cells.

\footnotetext{
${ }^{1}$ Department of Microbiology and Immunology, University of Minnesota, Minneapolis, MN, USA and ${ }^{2}$ Department of Lab Medicine and Pathology, University of Minnesota, Minneapolis, MN, USA

Correspondence: Gary M. Dunny (dunny001@umn.edu)
}

Received: 9 November 2016 Revised: 31 May 2017 Accepted: 9 June 2017

Published online: 30 June 2017 

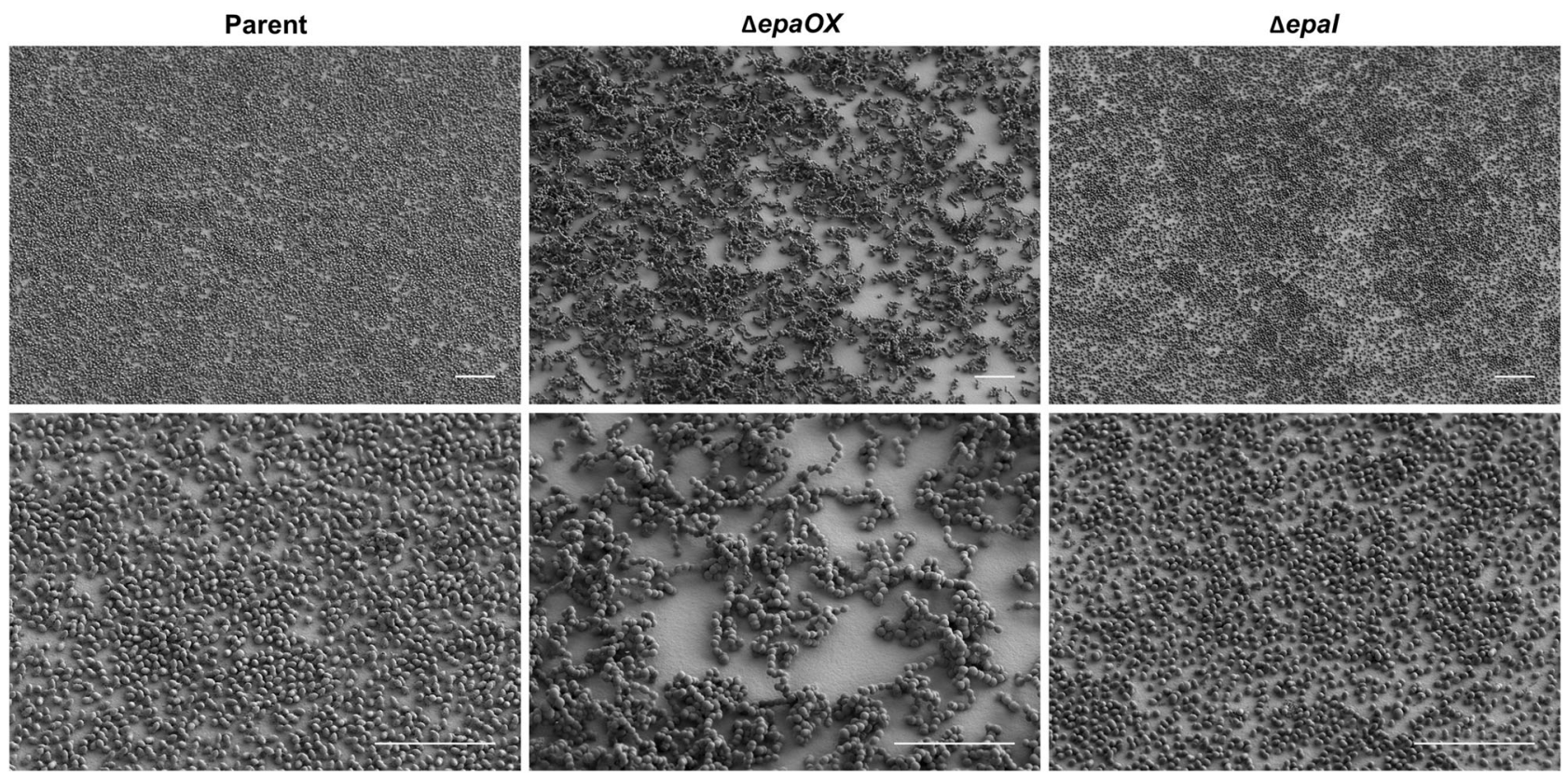

Fig. 1 Effects of epa deletions on E. faecalis biofilm formation. Scanning electron micrographs showing structural differences in biofilm architecture of parent strain OG1RF vs. isogenic epa mutants. Cultures were grown on Aclar membranes in CBRs for $24 \mathrm{~h}$ in the absence of antibiotics (scale bars, $10 \mu \mathrm{m}$ ). Representative images are shown from experiments performed a minimum of three times using biological replicates

\section{RESULTS}

epal and epaOX gene expression is unaffected by biofilm growth We reported previously that the GTFs epal and epaOX contributed to biofilm-associated antibiotic resistance using standard microtiter assays quantifying stained biofilm biomass as a measurement of biofilm growth. Deletion of either GTF resulted in wild-type biofilm formation in the absence of antibiotic and decreased nascent biofilm formation in the presence of subinhibitory concentrations of clinically relevant antibiotics. ${ }^{9}$ To initiate studies identifying the mechanism of biofilm-associated antibiotic resistance related to the epa genes, we considered whether there were differences in epal and epaOX gene expression in biofilm vs. planktonic populations. We hypothesized that if the epa genes were upregulated in biofilm cells, this could increase synthesis of the Epa polysaccharide and increase resistance to antibiotics. The results of quantitative reverse transcription PCR (RT-qPCR) revealed the relative fold-change in gene expression of epal (0.9 $+/-0.042)$ and epaOX $(1.0+/-0.002)$ was unchanged between biofilm and planktonic cell populations. These data indicate that differential epa gene expression in planktonic and biofilm populations is not responsible for biofilm-associated antibiotic resistance.

\section{Biofilm architecture is impacted by glycosyltransferases}

We sought to examine the effect of epa mutations on the architecture of $E$. faecalis biofilms considering that Epa cell-wall polysaccharides may affect surface properties, and may also contribute to the biofilm extracellular matrix. Parent and epa mutant strains were cultured on Aclar fluropolymer membranes in a continuous flow CDC biofilm reactor (CBR) for $24 \mathrm{~h}$ and biofilm architecture was assessed using scanning electron microscopy (SEM) (Fig. 1). The parent strain formed a monolayer biofilm, occasionally producing small microcolonies approximately two cells in depth. Deletion of epaOX resulted in production of a markedly different biofilm architecture. The $\triangle$ epaOX cells chained and clumped resulting in a complex 3D biofilm that was unable to completely cover the surface area of the Aclar membrane, which led to areas devoid of attached bacteria. Biofilms of the epal mutant were more similar in appearance to parent. Since the $\triangle e p a O X$ strain showed the most dramatic changes in biofilm architecture, we decided to examine further the differences between parent and $\triangle e p a O X$ biofilms and the effects of antibiotics on biofilm structure.

\section{E. faecalis restructures into microcolony biofilms in response to antibiotics}

To determine the impact of antibiotics on established biofilms, we cultured parent and $\triangle e p a O X$ strains in CBRs for $24 \mathrm{~h}$ without antibiotic and then exposed the resulting biofilms to antibiotic and examined architecture using SEM. The antibiotics used were chosen based on clinical relevance, and the concentrations were chosen based on previous results demonstrating that these concentrations were subinhibitory for planktonic growth. ${ }^{9}$ In the absence of antibiotics, parent cells formed monolayer biofilms that resulted in the formation of occasional microcolonies greater than two cells in depth after $48 \mathrm{~h}$ of growth. SEM micrographs showed that the biofilms of the $\triangle$ epaOX mutant remained the same throughout the $48 \mathrm{~h}$ time course with cells chaining, clumping, and forming complex 3D biofilms (Fig. 2). Both parent and $\triangle e p a O X$ biofilm cells appeared to form cellular appendages, or protrusions, that extended from the cells. The appendages remained over the time course of the experiment with subtle decreases in both strain backgrounds at $48 \mathrm{~h}$. In addition, parent and $\triangle e p a O X$ strains remained viable over the $48 \mathrm{~h}$ time course in the absence of antibiotic as observed using the immunofluorescence stain Syto9. Few cells stained with propidium iodide (PI), which stains dead cells or cells with increased permeability (Supplementary Fig. 1).

The most dramatic morphological changes in biofilm architecture appeared after parent biofilms were exposed to subinhibitory concentrations of antibiotic (Fig. 3 and Supplementary Fig. 2). After $8 \mathrm{~h}$ exposure to daptomycin (DAP), parent biofilm architecture changed drastically from monolayers into complex 3D architectures whereas $\triangle$ epaOX biofilm architecture remained 


\section{Biofilm development}

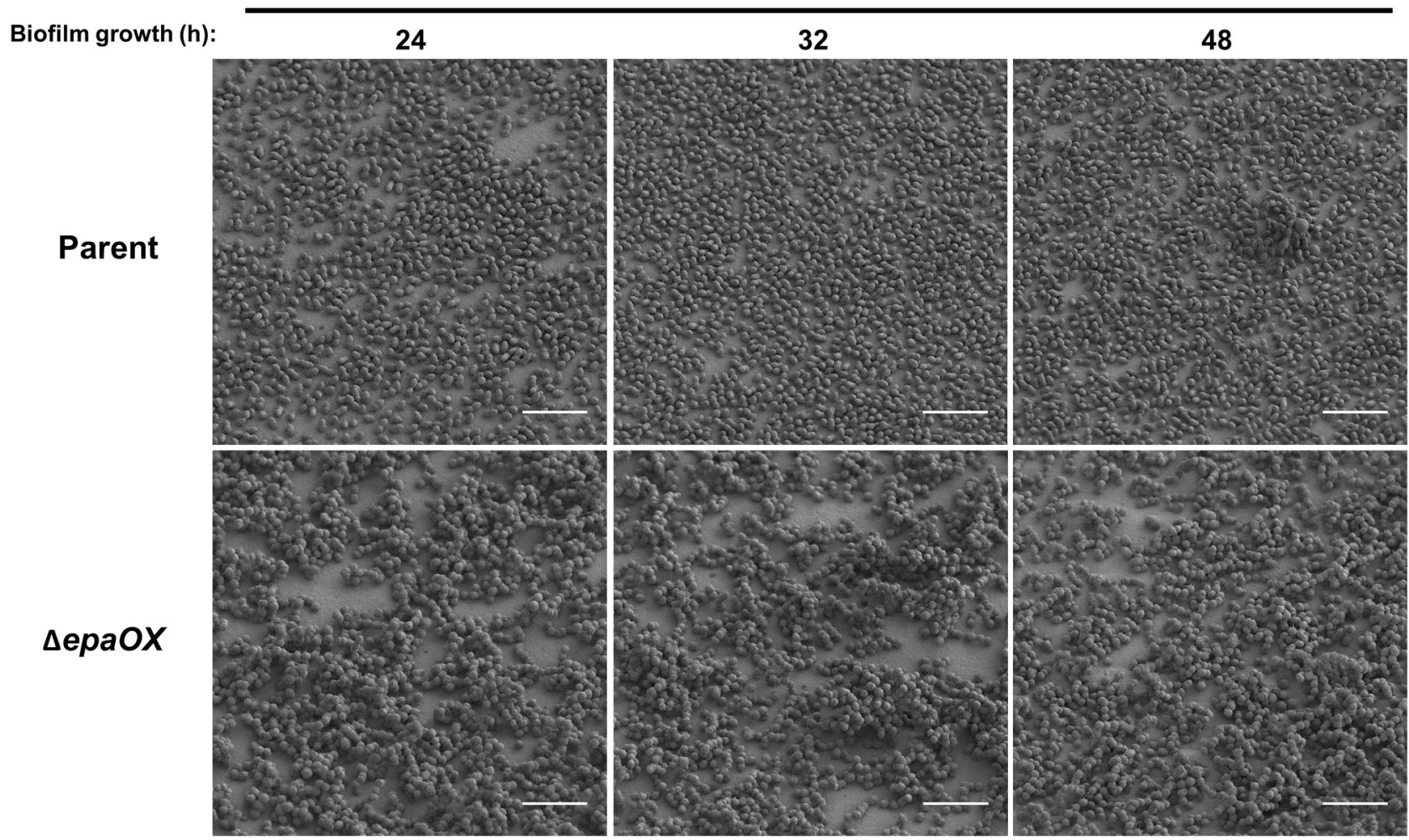

Fig. 2 Biofilm development of $E$. faecalis parent and $\triangle$ epaOX strains after extended cultivation. Experiments were performed as detailed in Materials and Methods. E. faecalis biofilms were cultured on Aclar membranes in CBRs for either 24, 32, or $48 \mathrm{~h}$ without antibiotic prior to fixation. The biofilms from each time point were visualized using low-voltage scanning electron microscopy to examine biofilm architecture (scale bars, $5 \mu \mathrm{m}$ ). Representative images are shown from experiments performed in triplicate with similar results

unchanged upon exposure to DAP. In addition, cell division of both parent and $\triangle e p a O X$ strains was affected with the most noticeable antibiotic-induced changes in cellular morphology occurring in the parent strain. DAP has been shown previously to affect cell division in $E$. faecalis and other bacterial species. ${ }^{17-19}$ Live/dead staining for both strains indicated increased uptake of $\mathrm{PI}$ at 8 and $24 \mathrm{~h}$ exposure to DAP suggestive of cell death (Supplementary Fig. 1). However, PI has the ability to also stain cells with increased permeability and extracellular DNA. Higher magnification SEM micrographs demonstrated the presence of extracellular material, which was visually distinct from the previous observation of cellular appendages, in the parent and $\triangle e p a O X$ strains (Fig. 4). These results suggest there is increased cell lysis and/or release of intracellular content from biofilm cells exposed to DAP, with somewhat higher levels of released material evident in the $\triangle$ epaOX biofilms (Fig. 4). Similar, albeit more subtle, restructuring of the parent biofilm occurred after exposure to gentamicin (Gm) (Supplementary Fig. 2). After $8 \mathrm{~h}$ exposure to $\mathrm{Gm}$, parent cells started clustering into small microcolonies that were more apparent by $24 \mathrm{~h}$. The $\triangle e p a O X$ mutant continued to form the same complex 3D architecture biofilms in the presence of $\mathrm{Gm}$ as was observed when cultured without antibiotics. There was no significant PI staining until parent and $\triangle e p a O X$ mutants had been exposed to $\mathrm{Gm}$ for $24 \mathrm{~h}$ (Supplementary Fig. 1). Higher magnification SEM micrographs further demonstrated microcolony formation and increases in regions of the surface devoid of adherent bacteria after $\mathrm{Gm}$ exposure in the parent strain. Interestingly, both parent and $\triangle e p a O X$ cells were devoid of cellular appendages when exposed to Gm (Supplementary Fig. 2b). In addition, $\triangle$ epaOX biofilms were susceptible to both DAP and $\mathrm{Gm}$ exposure as measured using quantitative counts indicating that both antibiotics were capable of interacting with the biofilm cells even though no perceptible architectural changes were observed (Supplementary Fig. 3). Since the parent biofilm restructuring could be explained by the attachment of planktonic cells into nascent microcolonies, we examined planktonic cell expansion in microtiter growth curve assays. Using culture medium identical to the CBR experiments $\left(10 \% \mathrm{TSB}^{-\mathrm{d}}\right.$ with or without antibiotics), there was no significant increase in the $\mathrm{OD}_{600}$ of parent or $\triangle e p a O X$ cells indicating no cell growth/expansion (data not shown). In total, these data suggest that parent restructuring from monolayers into microcolonies is mediated by the division or rearrangement of bacterial cells attached prior to antibiotic treatment vs. new colonization by planktonic cells in the CBR vessel.

Microcolony biofilms show reduced structural integrity relative to monolayer biofilms

While removing samples from the CBRs for imaging, we observed turbidity differences between parent and $\triangle e p a O X$ in the liquid phase of the CBR cultures following antibiotic exposure. This suggested that the structural stability of $E$. faecalis biofilms could be impacted by the complexity of the biofilm architecture, with increased turbidity resulting from detachment of biofilm cells. To further examine this possibility, we measured the optical density of buffer obtained after washing biofilm-coated membranes from the CBRs and recorded this value as relative detachment (Fig. 5). There was minimal detachment of parent cells from biofilms formed in the absence of antibiotics or in the presence of $\mathrm{Gm}$. However, there was a significant increase in detached cells when the parent biofilm became more structurally complex after exposure to DAP. In addition, $\triangle e p a O X$ biofilms were much less stable overall than parent biofilms in both the absence and 


\section{Daptomycin $(6 \mu \mathrm{g} / \mathrm{ml})$ effect on pre-formed biofilms}

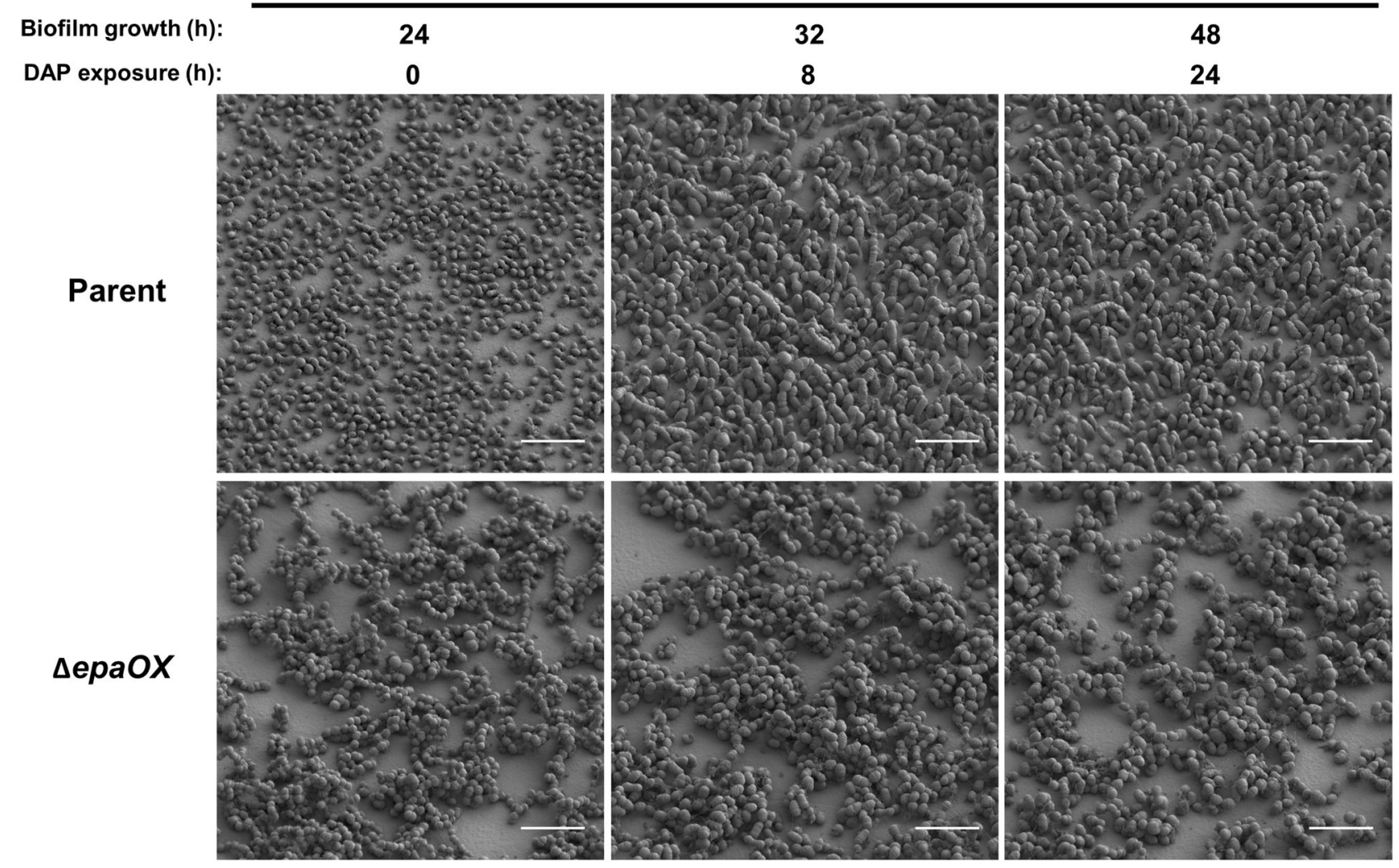

Fig. 3 Daptomycin effects on pre-formed E. faecalis biofilms. Experiments were performed as detailed in Materials and Methods. E. faecalis parent and $\triangle e p a O X$ strains were cultured on Aclar membranes for $24 \mathrm{~h}$ in CBRs without antibiotics to establish biofilms. Daptomycin (DAP) was then added to the culture medium at a concentration of $6 \mu \mathrm{g} / \mathrm{ml}$ and the effects on the pre-formed biofilms were assessed after 8 and 24 $\mathrm{h}$ of antibiotic exposure. Low-voltage scanning electron microscopy (SEM) was performed to examine biofilm architecture (scale bars, $5 \mu \mathrm{m}$ ). Representative images are shown from experiments performed in triplicate with similar results

presence of antibiotics. These data indicate that increased complexity in 3D architecture of the biofilm is associated with structural instability and increased susceptibility to antibiotics.

Variation in lectin-binding specificity and polysaccharide composition of $\triangle e p a O X$ is correlated with decreased biofilm integrity and antibiotic resistance

We used lectin-binding assays and biochemical analysis to examine the correlation between the cell-wall polysaccharide composition of $E$. faecalis strains and the corresponding biofilm architecture and antibiotic resistance. Out of eight total fluorescein-labeled lectins that were screened, Concanavalin A (ConA) was the only lectin that efficiently bound and stained $E$. faecalis biofilm cells. ConA bound strongly to parent biofilm cells with distinct staining of the cell envelope. However, only a subset of $\triangle$ epaOX biofilm cells interacted with ConA and the staining pattern was diffuse compared to parent (Fig. 6a). To determine how Epa polysaccharides could affect ConA binding, we submitted purified polysaccharides from parent and $\triangle$ epaOX strains for glycosyl composition analysis using combined gas chromatography/mass spectrometry (GC/MS) by acid methanolysis (Complex Carbohydrate Research Center, University of Georgia). Similar to previous reports, ${ }^{11,} 20$ our results demonstrate that rhamnose and glucose are the main polysaccharides detected in OG1RF and that the minor components of Epa are galactose, GalNAc, and GlcNAc (Fig. 6b). Deletion of epaOX resulted in similar amounts of rhamnose and glucose compared to parent, virtually undetectable amounts of galactose and GaINAc, and GlcNAc levels that deviated among replicates. The preferred sugar specificities for ConA are a-linked mannose and a-linked D-glucose, ${ }^{21}$ which are either nonexistent (mannose) or similar in abundance (glucose) between both the strains. These data suggest that ConA is interacting with a non-preferred sugar substrate that is more accessible on the parent biofilm cells, or that biofilm architecture itself impacts binding. Therefore, the composition of Epa polymers may influence the interaction between antibiotics and $E$. faecalis biofilm cells impacting biofilm-associated antibiotic resistance. However, antibiotic sequestration experiments using purified polysaccharide from parent and $\triangle e p a O X$ strains demonstrated no differential sequestration of DAP or Gm (Supplementary Fig. 4). These data instead suggest that antibiotic resistance is indirectly affected through Epa effects on biofilm architecture.

\section{DISCUSSION}

In some bacteria, elevated resistance to antibiotics in biofilms has been attributed to increased expression of genes whose products confer increased resistance. In Pseudomonas aeruginosa, enhanced expression of genes encoding diverse proteins including a DNA regulator, glucosyltransferase, efflux pump, type VI secretion component, putative two-component regulatory system, and hypothetical proteins have been linked to biofilm-induced resistance. ${ }^{6,22-26}$ Similarly, Escherichia coli biofilms are rendered more resistant to antibiotics as a result of increased regulation of multi-drug efflux pumps and a putative protein that affects cellwall composition. ${ }^{27}$ In this study, we determined that $E$. faecalis epal and epaOX gene expression is not increased in biofilms, 

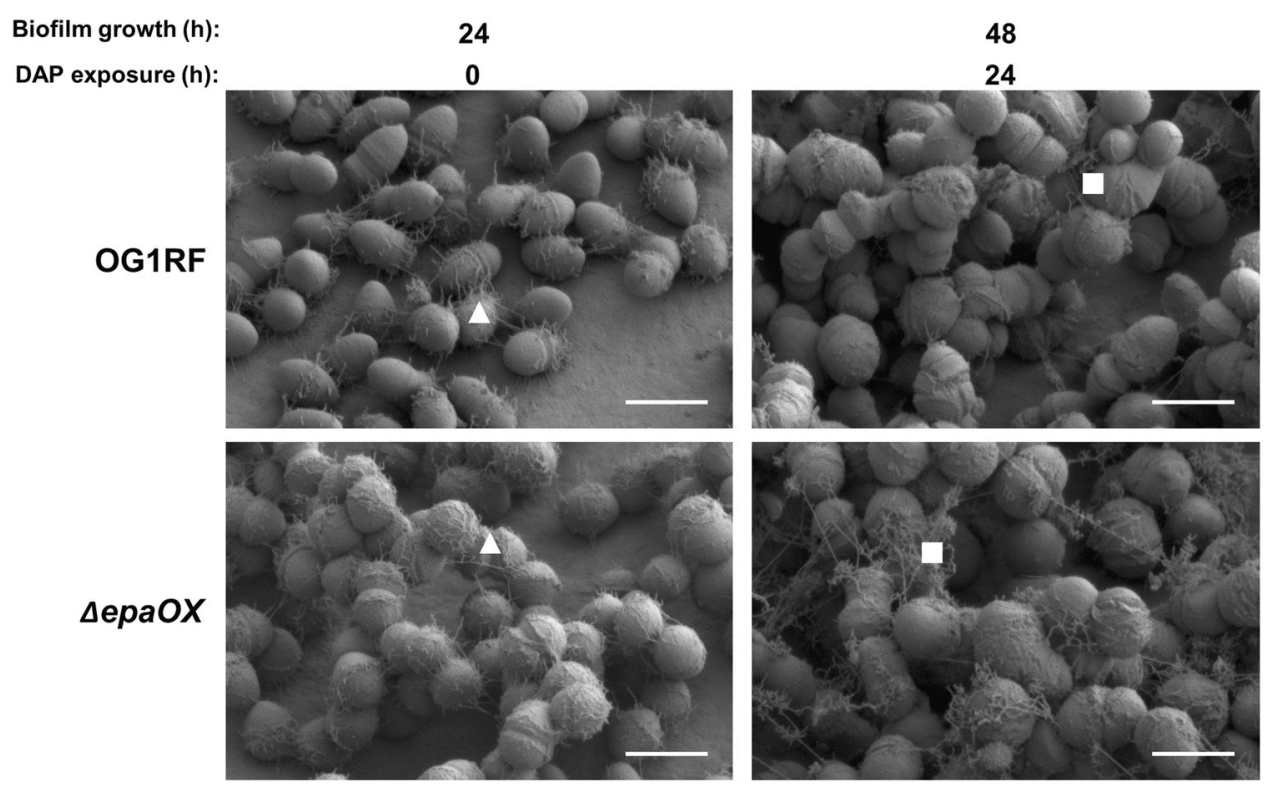

Fig. 4 Daptomycin exposed biofilms release extracellular material. Low-voltage scanning electron microscopy (SEM) demonstrating the presence of cellular appendages (triangles) produced by E. faecalis biofilms not exposed to antibiotics compared to extracellular debris (squares) released from biofilms exposed to daptomycin (DAP) (scale bars, $1 \mu \mathrm{m}$ )
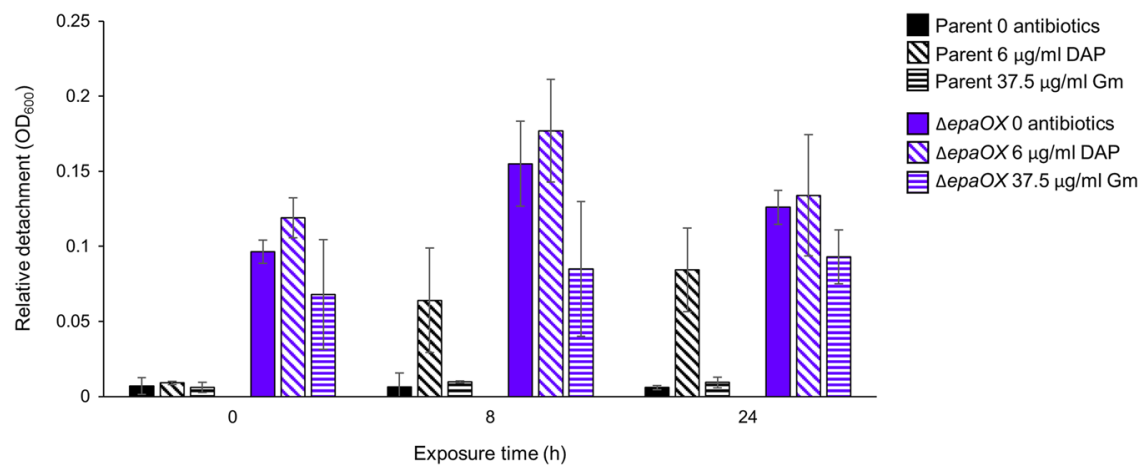

Fig. 5 E. faecalis biofilm stability. Relative detachment of biofilms cells from Aclar membranes after washing, as determined by measuring the $\mathrm{OD}_{600}$ of the wash buffer as described in the methods. Error bars represent standard deviation $(n=2$ for 0 antibiotics; $n=3$ for DAP and Gm)

arguing against increased expression as the mechanism for epamediated biofilm antibiotic resistance. However, our data do not exclude the possibility of other $E$. faecalis biofilm-associated resistance mechanisms that involve altered gene expression.

Another potential mechanism for increased antibiotic resistance within biofilm cells is altered interaction of antibiotics with their target sites as a result of changes in the cell envelope. To begin to address this question, we screened a panel of fluorescein-labeled lectins for the ability to bind parent and $\triangle e p a O X$ biofilm cells. Our data showed that the lectin ConA bound to parent, but not $\triangle e p a O X$ biofilm cells. These data suggest that a $\triangle e p a O X$ mutant could also exhibit altered interaction with antibiotics due to changes in surface properties, permeability, alteration of an antibiotic target, or differences in sequestration of antibiotic by polysaccharide. We showed previously altered polysaccharide production by the $\triangle$ epaOX mutant. ${ }^{9}$ Polysaccharide components of the biofilm matrix have been reported in $P$. aeruginos $a$ and in the yeast Candida albicans to directly sequester antimicrobials, and these organisms also produce increased amounts of cyclic glucans within biofilm cells, which can bind antimicrobials to prevent access to their target sites. ${ }^{6,28}$ Epa polysaccharides could also sequester antibiotics within the $E$. faecalis biofilms. However, we found that purified polysaccharides from parent and $\triangle$ epaOX strains did not differentially bind DAP or Gm arguing against antibiotic sequestration as the mechanism of epa-mediated biofilm-associated antibiotic resistance.

The GTF genes we analyzed are localized to the epa gene cluster, which includes the epaA-R genes ${ }^{11,} 12$ and additional downstream genes including epaOX.9, 29 There are seven predicted GTFs within the 18 annotated epa gene cluster and three additional predicted GTFs (including epaOX) in the OG1RF region immediately downstream of epaR. ${ }^{29}$ Previous reports have suggested that EpaB is a rhamnosyltransferase that helps construct the Epa backbone. ${ }^{11}$ Our data show that the Epa rhamnopolysaccharide was deficient in galactose and GalNAc upon deletion of epaOX suggesting a role in Epa decoration. The V583-associated gene epaX was also shown to help decorate $\mathrm{Epa} ;{ }^{10}$ however, we determined no functional redundancy to the OG1RF epaOX GTF (data not shown). These cumulative results have provided some functional characterization of the epa GTFs. Our current and future efforts will focus on the impact of antibiotics on Epa composition in biofilm cells. We have preliminary data demonstrating that the Epa content is similar between untreated and treated planktonic and biofilm cells (Supplementary Fig. 5). However, the exact composition of each monosaccharide may differ; therefore, we aim to perform largescale cultivation experiments to obtain adequate quantities of polysaccharide for complete biochemical analysis. 
a.
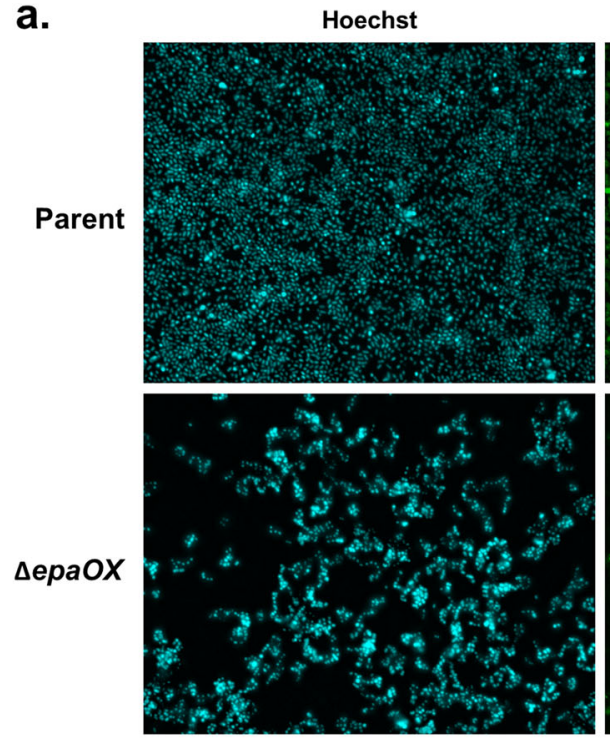

ConA
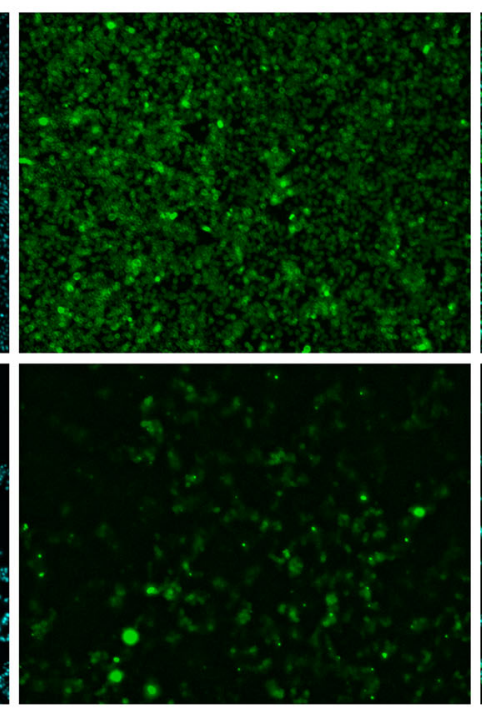

Merge
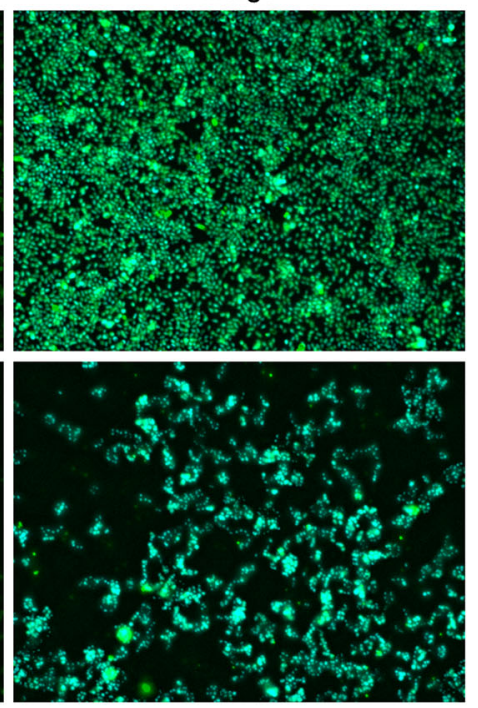

b.

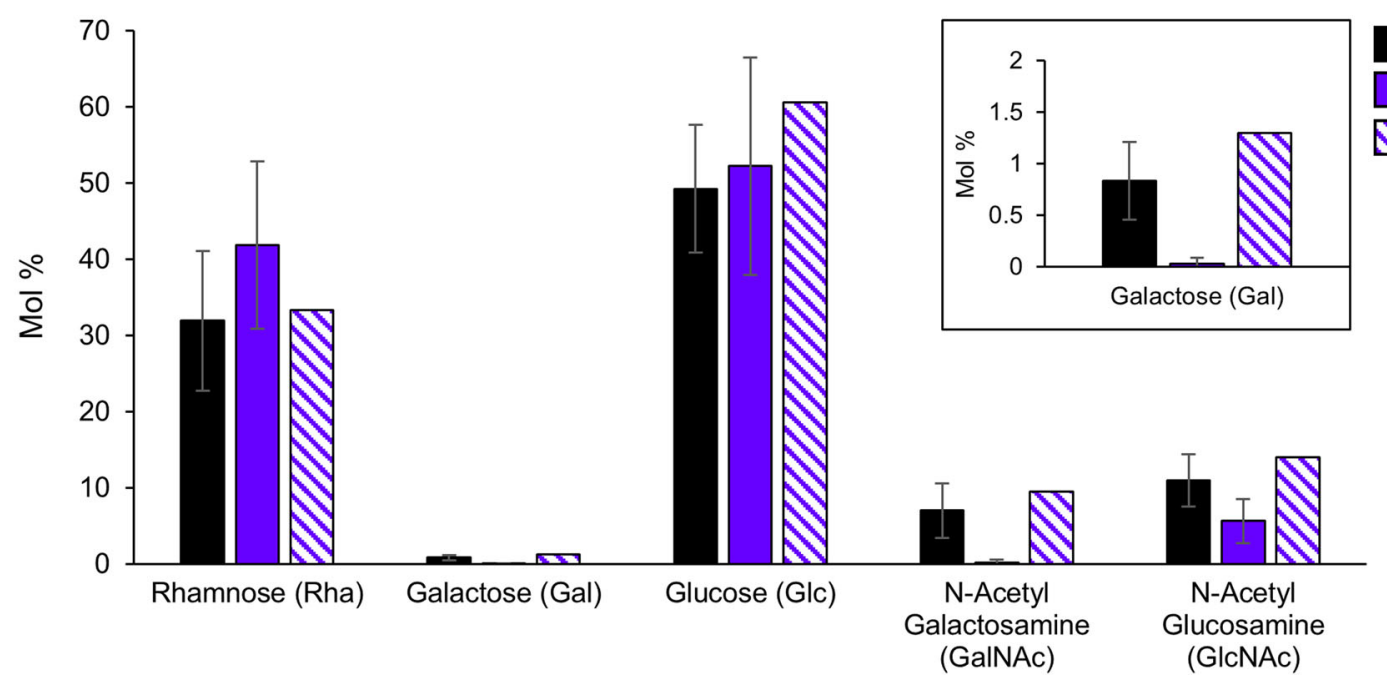

Fig. 6 Deletion of epaOX affects lectin-binding ability and polysaccharide composition. a Immunofluorescence micrographs of parent and $\Delta$ epaOX biofilms. E. faecalis cells were cultured on Aclar membranes in CBRs for $24 \mathrm{~h}$ and stained with the DNA label Hoechst 33342 (blue) and the fluorescein-labeled lectin concanavalin A (ConA-green). Representative images at a magnification of 100x are shown from experiments performed in triplicate. b Carbohydrate composition of purified polysaccharides obtained from parent, $\triangle e p a O X$, and $\triangle e p a O X:: e p a O X$ strains. E. faecalis strains were cultured to exponential phase for polysaccharide extraction. Error bars represent standard deviation $(n=3$ for parent, $\Delta$ epaOX; $n=1$ for $\Delta$ epaOX::epaOX)

The Epa polysaccharides are predicted to be buried within the $E$. faecalis cell wall. ${ }^{20} \mathrm{We}$ and others have demonstrated that deletion or disruption of epa genes results in aberrations in cellular morphology. ${ }^{9-11}$ Since Epa appears integral in maintaining cell shape, we investigated its role in biofilm structure. Our data shows that deletion of epaOX markedly affects biofilm architecture. The $\triangle e p a O X$ cells chain, clump, and form ultrastructured microcolonies compared to the monolayer biofilms formed by parent and the $\Delta e p a l$ mutant. This drastic architectural change resulting from deletion of epaOX led us to hypothesize that biofilm architecture may play an important, intrinsic role in biofilmassociated antibiotic resistance.

To determine if biofilm architecture directly impacts antibiotic resistance, we analyzed biofilm development in the absence and presence of subinhibitory DAP and Gm concentrations using SEM. Surprisingly, it was the parent biofilm architecture that drastically changed after exposure to DAP or Gm, resembling $\triangle$ epaOX biofilm architecture in the absence of antibiotics, which remained relatively unchanged following antibiotic challenge. Parent cells formed a monolayer biofilm in the absence of antibiotics with sporadic microcolony formation only after $48 \mathrm{~h}$ of growth. However, upon exposure to subinhibitory concentrations of DAP parent biofilm cells rapidly reorganized into highly structured microcolonies reminiscent of $\triangle e p a O X$ biofilms. Microcolony formation of parent cells in the presence of $\mathrm{Gm}$ was more subtle, but readily apparent (Supplementary Fig. 2).

This is not the first report demonstrating reorganization of bacterial biofilms. Moormeier et al. ${ }^{30}$ showed that Staphylococcus aureus biofilms change their matrix composition in order to transition from a multiplication stage to exodus. During the exodus stage, a subpopulation of biofilm cells detach and tower structures of robust growth develop at distinct foci. ${ }^{30} \ln P$. aeruginosa multiple growth conditions and regulatory factors contribute to microcolony development; ${ }^{31-35}$ however, the extent to which microcolony formation results from rearrangement of monolayers is unclear. 
The function of microcolonies in regard to environmental survival or virulence is likely to vary depending on the species and growth conditions. It has been proposed that $S$. aureus tower biofilms are important for pathogenesis enabling the bacteria to detach and disseminate to additional infection sites. ${ }^{7,36,37}$ In $P$. aeruginosa, the prototypical mushroom-shaped biofilm microcolonies are more resistant to detergent and antibiotics than less structured biofilms produced by certain mutants. ${ }^{38,39}$ We showed that transition of $E$. faecalis biofilm structure from monolayers to microcolonies resulted in decreased stability and enhanced biofilm detachment. The $\triangle$ epaOX biofilm demonstrated the greatest extent of microcolony development and highest amount of cellular detachment in the presence and absence of antibiotics, while parent biofilms that restructured from monolayers to microcolonies in the presence of DAP also showed a high degree of cellular detachment. We have also shown that two independent clinical isolates (FVE12, ${ }^{40}$ MMH594 ${ }^{41}$ ) exhibit increased relative detachment and cell death/permeability of biofilm cells after exposure to DAP suggesting antibiotic-induced biofilm instability is common among $E$. faecalis species (Supplementary Fig. 6). While increased biofilm detachment could be advantageous for bacterial dissemination during infection, detachment could be disadvantageous for $E$. faecalis by making the detached bacteria more susceptible to antibiotics or the immune response.

Microcolony formation in CBR grown $E$. faecalis biofilms could be viewed as a stress response where the source of stress for parent biofilms is antibiotics, while $\triangle e p a O X$ mutant cells may be experiencing increased stress in the absence of antibiotics due to cell envelope defects. In support of this hypothesis, RT-qPCR analysis demonstrated that $E$. faecalis parent biofilms treated with DAP or Gm showed increased expression of three stress response genes (sigV, croR, and liaR) compared to untreated biofilms (Supplementary Table 2). These three stress response genes show increased expression in response to environmental pressure, cellwall active antibiotics, and cell envelope damage, ${ }^{42-45}$ respectively, in E. faecalis and other organisms. Interestingly, Gmexposed parent biofilms exhibited the largest fold-change increase in the stress response genes even though DAP-exposed parent biofilms demonstrated the most drastic biofilm architecture phenotypes. When expression of these three genes in biofilm cells of untreated parent and $\triangle e p a O X$ was compared, there was a modest increase in liaR expression in $\triangle$ epaOX biofilms and little effect on $c r o R$ or sigV transcription. However, it is possible the $\triangle e p a O X$ mutant could be experiencing cell envelope stress during both planktonic and biofilm growth, complicating interpretation of analysis. We are currently developing an inducible gene-specific knockdown system that may enable induction of the $\triangle$ epaOX cell envelope defects during biofilm growth to better address this issue.

Interestingly, similar studies examining the transcriptome of antibiotic treated biofilms has been performed in Klebsiella pneumoniae. Van Laar et al. ${ }^{46}$ exposed preformed $K$. pneumoniae biofilms to sublethal, but not subinhibitory, concentrations of the carbapenem antibiotic imipenem and determined that there was differential regulation of the stress response with some genes being upregulated while others were downregulated. The authors also demonstrated that imipenem exposure resulted in a reversible morphological aberration in cell size, shape, and appearance of additional extracellular material, not unlike the phenotypes we have observed herein for $E$. faecalis antibiotic exposure. These complementary experimental approaches performed in two distinct organisms highlight the potentially congruent pathways employed by bacteria to sense and respond to environmental insult.

E. faecalis is an important pathogen in HAls and understanding the mechanisms that increase antibiotic resistance to this recalcitrant microbe are of extreme importance. Our work provides insight into the role of Epa polysaccharides in biofilm architecture. In addition, our data identified conditions where $E$. faecalis biofilms with increased 3D complexity displayed decreased structural stability. Currently, our highest priority is to elucidate the mechanism of antibiotic-induced rearrangement from monolayer to microcolony biofilms. Our data suggests that this transition occurs by division and possibly by rearrangement of the adherent biofilm cells in the monolayers rather than by recruitment of new cells from the planktonic phase. We are initiating single cell analysis of adherent cells during the transition to address this issue. The current data also provide rationale for future experiments to determine the transcriptional response of $E$. faecalis biofilms to DAP and Gm on a genome-wide level. RNAseqbased identification of the most highly induced genes will enable design of genetic screens to identify the critical sensors and effectors of the changes in biofilm architecture induced by each antibiotic. Ultimately, a better understanding of the role of the cellular architecture of $E$. faecalis biofilms in antibiotic resistance may inform improved strategies to treat or prevent recalcitrant infections involving enterococcal biofilms.

\section{MATERIALS AND METHODS}

Bacterial strains and growth conditions

E. faecalis strains used in this study include the parent strain $\mathrm{OG}_{\mathrm{RF}}{ }^{47}$ and isogenic derivatives $\triangle e p a O X$ (JD102), $\triangle$ epal (JD106), and $\triangle$ epaOX::epaOX (JD102[pJLD4]). ${ }^{9}$ E. faecalis strains were cultured overnight at $37^{\circ} \mathrm{C}$ in brain heart infusion (BHI) medium (Becton, Dickinson and Co., Franklin Lakes, NJ) using static conditions. Tryptic soy broth without dextrose (TSB ${ }^{-\mathrm{d}}$ ) (Becton, Dickinson and Co.) was used for all other experiments. Antibiotics were added to the medium as appropriate: DAP (Biotang Inc., Lexington, MA) at $6 \mu \mathrm{g} / \mathrm{ml}$ with $50 \mathrm{mg} / \mathrm{l} \mathrm{CaCl}$, gentamicin at $37.5 \mu \mathrm{g} / \mathrm{ml}$, spectinomycin at $1000 \mu \mathrm{g} / \mathrm{ml}$, erythromycin at $10 \mu \mathrm{g} / \mathrm{ml}$, or nisin at $25 \mathrm{ng} / \mathrm{ml}$. All antibiotics were purchased from Sigma-Aldrich (St Louis, MO) unless stated otherwise. The number of replicates performed for each experiment are included in the figure legends and table footnotes.

\section{RNA purification and RT-qPCR}

E. faecalis biofilm and planktonic cells were obtained from CBRs that were set-up as described below. To compare epal and epaOX gene expression, polycarbonate coupons were removed aseptically after $24 \mathrm{~h}$ incubation and placed in six-well plates (four coupons/well) containing $5 \mathrm{ml}$ distilled water and incubated for $5 \mathrm{~min}$ at room temperature to remove nonadherent cells. Aclar films were placed in $50 \mathrm{ml}$ conical tubes (four Aclar films/tube) containing $30 \mathrm{ml}$ KPBS and inverted to remove non-adherent cells. To obtain attached biofilm cells, twelve coupons and four Aclar were placed per $50 \mathrm{ml}$ conical tube containing $8 \mathrm{ml}$ KPBS supplemented with 2 $\mathrm{mM}$ EDTA and $16 \mathrm{ml}$ of RNAprotect (Qiagen, Valencia, CA) and vortexed at $4^{\circ} \mathrm{C}$ for $5 \mathrm{~min}$. Vortexed biofilm cells were transferred to a new conical tube and centrifuged $(6640 \times g)$ at $4{ }^{\circ} \mathrm{C}$ for $15 \mathrm{~min}$ for RNA extraction. Four milliliters of planktonic culture was removed from the reactor vessel and incubated with two volumes RNAprotect. After $5 \mathrm{~min}$ incubation at room temperature, planktonic cells were pelleted.

To compare stress response gene expression, Aclar films were removed after $8 \mathrm{~h}$ exposure to either DAP or $\mathrm{Gm}$ and washed as described above to remove non-adherent cells. To obtain attached biofilm cells, Aclar films were submerged in $4 \mathrm{ml}$ KPBS supplemented with $2 \mathrm{mM}$ EDTA and physically scraped off the film using one sterile razor blade per Aclar film. The scraped biofilm cell suspension was incubated with $8 \mathrm{ml}$ RNAprotect for $5 \mathrm{~min}$ at room temperature and pelleted. One-hundred milliliters of planktonic culture was removed from the reactor vessel and pelleted. Insufficient quantities of planktonic culture were obtained from CBRs containing $\mathrm{Gm}$; therefore, parent cells were cultured in batch using TSB $^{-\mathrm{d}}$ with or without $37.5 \mu \mathrm{g} / \mathrm{ml} \mathrm{Gm}$. Four-milliliters of planktonic culture was harvested at an $\mathrm{OD}_{600}$ of $\sim 0.5$ and incubated with $8 \mathrm{ml}$ RNAprotect. After 5 min incubation at room temperature, planktonic cells were pelleted.

Biofilm and planktonic cells were lysed enzymatically using $30 \mathrm{mg} / \mathrm{ml}$ lysozyme and $500 \mathrm{U} / \mathrm{ml}$ mutanolysin in TE $(10 \mathrm{mM}$ Tris, pH8; $1 \mathrm{mM}$ EDTA, pH8) for $10 \mathrm{~min}$ at $37^{\circ} \mathrm{C}$. Following lysis, RNA was extracted using the RNeasy mini kit (Qiagen) following the manufacturers protocol. One to four micrograms of total RNA was subjected to Turbo DNase treatment using the rigorous method as described (Ambion/ThermoScientific, Waltham, MA). Complementary DNA was prepared following the SuperScript III first- 
strand synthesis system for RT-PCR kit (Invitrogen/ThermoScientific) using random hexamers. Conventional PCR using universal $16 \mathrm{~S}$ primers was used to verify DNA removal. RT-qPCR was performed using SYBR green master mix and an iCycler iQ5 (Bio-rad, Hercules, CA). Total reaction volumes per well were $25 \mu \mathrm{l}$ containing $200 \mathrm{nM}$ of each primer. Primer pairs and melting temperature $(\mathrm{Tm})$ used for RT-qPCR are found in Supplementary Table 1. Three-step RT-qPCR parameters were as follows: 3 min at $95^{\circ} \mathrm{C}$ (one cycle); $10 \mathrm{~s}$ at $95^{\circ} \mathrm{C}, 30 \mathrm{~s}$ at $53^{\circ} \mathrm{C}$ or $59.5^{\circ} \mathrm{C}, 30 \mathrm{~s}$ at $75^{\circ} \mathrm{C}$ (40 cycles); $50^{\circ}-95^{\circ} \mathrm{C}$ melt curve with $0.5^{\circ}$ temperature change. The primer efficiencies and cycle threshold $(C \mathrm{t})$ measurements were calculated by the iQ5 Optical System Software version 2.1 (2009; Bio-Rad). All experiments met the following criteria: primer efficiency $78-100 \% ; r^{2} \geq 0.995$; and $\mathrm{Ct}$ for no-RT control samples $>35$. The Pfaffl method was used to analyze data and calculate relative fold-change normalized to relA (Pfaffl 2001). RT-qPCR was performed on technical replicates from two biological samples of biofilm and planktonic cells.

\section{Polysaccharide purification and glycosyl composition analysis}

E. faecalis parent, isogenic mutants, and complemented strains were cultured in $500 \mathrm{ml} \mathrm{TSB}$ containing appropriate antibiotics, if necessary for plasmid maintenance, at $37^{\circ} \mathrm{C}$ under static conditions to an $\mathrm{OD}_{600}$ of

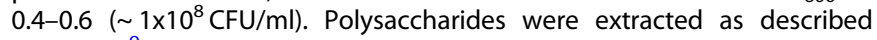
previously ${ }^{9}$ with modifications. Briefly, pelleted cells were washed with 10 $\mathrm{ml}$ of sucrose solution ( $25 \%$ sucrose, $10 \mathrm{mM}$ Tris- $\mathrm{HCl} \mathrm{pH} \mathrm{8),} \mathrm{resuspended} \mathrm{in}$ $15 \mathrm{ml}$ sucrose solution supplemented with $1 \mathrm{mg} / \mathrm{ml}$ lysozyme and $10 \mathrm{U} / \mathrm{ml}$ mutanolysin and incubated with gentle agitation at $37^{\circ} \mathrm{C}$ overnight. Supernatant fractions were collected from pelleted cells and treated with $200 \mu \mathrm{g} / \mathrm{ml} \mathrm{RNase} \mathrm{A,} 20 \mathrm{U} / \mathrm{ml}$ DNase, $5 \mathrm{mM} \mathrm{MgCl}$, and $1 \mathrm{mM} \mathrm{CaCl} 2$ at $37^{\circ} \mathrm{C}$ for $8 \mathrm{~h}$. Proteinase $\mathrm{K}$ was added at $50 \mu \mathrm{g} / \mathrm{ml}$ to the supernatant and incubated at $37^{\circ} \mathrm{C}$ overnight. Remaining impurities were extracted using $500 \mu \mathrm{l}$ chloroform. The aqueous phase was transferred to a new tube following centrifugation $(6640 \times g)$ for $15 \mathrm{~min}$. Polysaccharides were precipitated by adding ethanol to a final concentration of $75 \%$ and incubation at $-70^{\circ} \mathrm{C}$ for $30 \mathrm{~min}$ followed by $60 \mathrm{~min}$ centrifugation $(6640 \times \mathrm{g})$ at $4{ }^{\circ} \mathrm{C}$. Precipitated pellets were washed using $75 \%$ ethanol and allowed to air dry. Polysaccharide samples were submitted for glycosyl composition analysis to the Complex Carbohydrate Research Center (University of Georgia), which performed combined GC/MS using acidic methanolysis.

\section{Biofilm growth}

Biofilms were cultured in CBRs on Aclar fluoropolymer film (Electron Microscopy Sciences, Hatfield, PA) and polycarbonate coupons (BioSurface Technologies Corp., Bozeman, MT). The CBR was assembled using 100\% $\mathrm{TSB}^{-\mathrm{d}}$ in the reactor vessel that contained eight Aclar films secured by polypropylene rods containing polycarbonate coupons. The vessel was placed on a stir plate rotating at $130 \mathrm{rpm}$ and was attached via peristaltic pump tubing to a carboy containing $10 \%$ TSB $^{-d}$ continuous flow medium. The entire CBR assembly was placed in a $37^{\circ} \mathrm{C}$ room. Two-milliliters of overnight culture at a cell density of approximately $1 \times 10^{9} \mathrm{CFU} / \mathrm{ml}$ was used to inoculate the CBR vessel. To allow bacterial attachment, the inoculated CBR was run for $4 \mathrm{~h}$ in batch culture at $130 \mathrm{rpm}$ before starting the peristaltic pump at a flow rate of $8 \mathrm{ml} / \mathrm{min}$ for $20 \mathrm{~h}$ to enable a continuous flow of medium. After $24 \mathrm{~h}$, the CBR carboy was exchanged for fresh $10 \% \mathrm{TSB}^{-\mathrm{d}}$ medium supplemented with DAP, gentamicin, or no antibiotics and incubated with a flow rate of $8 \mathrm{ml} / \mathrm{min}$ for $24 \mathrm{~h}$. Samples were removed and rinsed in $30 \mathrm{ml}$ KPBS at the following time points: $24 \mathrm{~h}$ ( $0 \mathrm{~h}$ antibiotic exposed), $32 \mathrm{~h}$ ( $8 \mathrm{~h}$ antibiotic exposed), and $48 \mathrm{~h}(24 \mathrm{~h}$ antibiotic exposed). Rinsed Aclar membranes were prepared for microscopy as described below. To estimate the relative detachment of biofilm cells, $1 \mathrm{ml}$ from the $30 \mathrm{ml}$ rinse suspension was measured at an $\mathrm{OD}_{600}$ at the designated time points.

\section{Immunofluorescence microscopy}

Biofilms cultured on Aclar film were rinsed $3 \times$ using KPBS and stained for $15 \mathrm{~min}$ at room temperature with either $5 \mu \mathrm{g} / \mathrm{ml}$ Hoechst 33,342 and 20 $\mathrm{ng} / \mathrm{\mu l}$ fluorescein-labeled Concanavalin A (Vector Laboratories, Burlingame, (A), or $2.5 \mu \mathrm{M}$ Syto 9 and $16 \mu \mathrm{M} \mathrm{PI}$. Other lectins tested include fluoresceinlabeled Soybean (SBA), wheat germ (weak interaction with parent and $\triangle$ epaOX [WGA]), Dolichos biflorus (DBA), Ulex europaeus (UEA 1), Ricinus communis $\left(\mathrm{RCA}_{120}\right)$, Peanut (PNA), and Banana lectin (weak interaction with parent [BanLec]). All stains were purchased from Molecular Probes/ ThermoFisher Scientific unless stated otherwise. Immunolabeled samples were either directly visualized by wet-mount or fixed in $2 \%$ paraformaldehyde in KPBS overnight at room temperature and mounted. Prolong diamond antifade mountant (ThermoFisher Scientific) was added to fixed Aclar on slides with a cover glass spacer to avoid damaging the biofilm structure (SecureSeal Adhesive, Electron Microscopy Sciences). Images were captured with a Zeiss AX10 using Zen 2.1 software as a widefield snapshot or $z$-stack with a $20 \times 0.8$ numerical aperture (NA) or $100 \times$ $1.3 \mathrm{NA}$ objective. The images presented are maximum intensity projections obtained using the Fiji ImageJ package. ${ }^{48}$

\section{Scanning electron microscopy (SEM)}

Reagents were purchased from Electron Microscopy Sciences (Hatfield, PA) unless otherwise noted. Samples were prepared using the cationic dye stabilization methods originally developed by Erlandsen et al. ${ }^{49}$ and modified as we have reported previously..$^{50}$ Briefly, the Aclar films were rinsed $3 \times$ with KPBS and fixed (primary fixation) for $22 \mathrm{~h}$ in cacodylatebuffered solution $(135 \mathrm{mM})$ containing methanol-free, EM-grade $2 \%$ formaldehyde and $2 \%$ glutaraldehyde, $4 \%$ sucrose, and $0.15 \%$ Alcian Blue 8GX (Sigma-Aldrich). Primary fixative was removed, Aclar coupons were rinsed $3 \times$ with sodium cacodylate buffer, and fixed (secondary fixation) for $1 \mathrm{~h}$ in a cacodylate-buffered solution $(150 \mathrm{mM})$ containing $1 \%$ osmium tetroxide and $1.5 \%$ potassium ferrocyanide. Fixed samples were chemically dried using a graded ethanol series $(25,50,70,85,95$ [2x], 100\% [2x]), processed in a $\mathrm{CO}_{2}$-based critical point dryer (tousimis, Rockville, $\mathrm{MD}$ ), and sputter coated with a 1- to 2-nm layer of iridium (Leica EM ACE600, Buffalo Grove, IL). Low-voltage SEM imaging $(0.8 \mathrm{kV})$ was performed using a Hitachi SU8230 field-emission instrument with the low-angle backscatter and secondary electron detectors.

\section{Data availability}

All data that support the findings of this study are available from the corresponding author upon reasonable request.

\section{ACKNOWLEDGEMENTS}

This work was supported by PHS Grants Al58134 and Al122742 to G.M.D, and grant T90 DE0227232 awarded to J.L.D. from the National Institute of Dental and Craniofacial Research. Parts of this work were carried out in the Characterization Facility (CharFac) at the University of Minnesota, which receives partial support from the NSF through the Materials Research Science and Engineering Centers program. The Hitachi SU8230 SEM was provided by NSF MRI DMR-1229263. Glycosyl composition analysis was supported by the Chemical Sciences, Geosciences and Biosciences Division, Office of Basic Energy Sciences, U.S. Department of Energy grant (DE-FG02-93ER20097) to Parastoo Azadi at the Complex Carbohydrate Research Center. The content is solely the responsibility of the authors and does not necessarily represent the official views of the National Institute of Dental \& Craniofacial Research, the NIH, NSF, or CCRC.

\section{AUTHOR CONTRIBUTIONS}

J.L.D. designed and performed experiments, acquired and analyzed data, and drafted the manuscript. J.L.N. assisted in sample preparation for microscopy. A.M.T.B. provided microscopy technical guidance and data analysis. G.M.D. assisted in data analysis and manuscript revision.

\section{ADDITIONAL INFORMATION}

Supplementary Information accompanies the paper on the npj Biofilms and Microbiomes website (doi:10.1038/s41522-017-0023-4).

Competing interests: The authors declare that they have no competing financial interests.

Publisher's note: Springer Nature remains neutral with regard to jurisdictional claims in published maps and institutional affiliations.

\section{REFERENCES}

1. Sievert, D. M. et al. Antimicrobial-resistant pathogens associated with healthcareassociated infections: summary of data reported to the National healthcare safety network at the centers for disease control and prevention, 2009-2010. Infect. Control Hosp. Epidemiol. 34, 1-14 (2013).

2. Hoyle, B. D. \& Costerton, J. W. Bacterial resistance to antibiotics: the role of biofilms. Prog. Drug. Res. 37, 91-105 (1991). 
3. Potera, C. Forging a link between biofilms and disease. Science 283, 1837-1839 (1999).

4. Lewis, K. Multidrug tolerance of biofilms and persister cells. Curr. Top. Microbiol. Immunol. 322, 107-131 (2008).

5. Mah, T. F. \& O'Toole, G. A. Mechanisms of biofilm resistance to antimicrobial agents. Trends Microbiol. 9, 34-39 (2001).

6. Mah, T. F. et al. A genetic basis for Pseudomonas aeruginosa biofilm antibiotic resistance. Nature 426, 306-310 (2003).

7. Donlan, R. M. \& Costerton, J. W. Biofilms: survival mechanisms of clinically relevant microorganisms. Clin. Microbiol. Rev. 15, 167-193 (2002).

8. Fernandez Guerrero, M. L., Goyenechea, A., Verdejo, C., Roblas, R. F. \& de Gorgolas, M. Enterococcal endocarditis on native and prosthetic valves: a review of clinical and prognostic factors with emphasis on hospital-acquired infections as a major determinant of outcome. Medicine (Baltimore). 86, 363-377 (2007).

9. Dale, J. L., Cagnazzo, J., Phan, C. Q., Barnes, A. M. \& Dunny, G. M. Multiple roles for Enterococcus faecalis glycosyltransferases in biofilm-associated antibiotic resistance, cell envelope integrity, and conjugative transfer. Antimicrob. Agents Chemother. 59, 4094-4105 (2015).

10. Rigottier-Gois, L. et al. The surface rhamnopolysaccharide Epa of Enterococcus faecalis is a key determinant of intestinal colonization. J. Infect. Dis. 211, 62-71 (2015).

11. Teng, F., Singh, K. V., Bourgogne, A., Zeng, J. \& Murray, B. E. Further characterization of the epa gene cluster and Epa polysaccharides of Enterococcus faecalis. Infect. Immun. 77, 3759-3767 (2009).

12. Xu, Y., Murray, B. E. \& Weinstock, G. M. A cluster of genes involved in polysaccharide biosynthesis from Enterococcus faecalis OG1RF. Infect. Immun. 66 4313-4323 (1998).

13. Singh, K. V., Lewis, R. J. \& Murray, B. E. Importance of the epa locus of Enterococcus faecalis OG1RF in a mouse model of ascending urinary tract infection. J. Infect. Dis. 200, 417-420 (2009).

14. Xu, Y., Singh, K. V., Qin, X., Murray, B. E. \& Weinstock, G. M. Analysis of a gene cluster of Enterococcus faecalis involved in polysaccharide biosynthesis. Infect. Immun. 68, 815-823 (2000).

15. Ocvirk, S. et al. Surface-associated lipoproteins link Enterococcus faecalis virulence to colitogenic activity in IL-10-deficient mice independent of their expression levels. PLoS Pathog. 11, e1004911 (2015).

16. Hoff, J. S. \& Kristich, C. J. Thymidylate limitation potentiates cephalosporin activity toward Enterococci via an Exopolysaccharide-based mechanism. ACS Chem. Biol. 11, 1561-1568 (2016).

17. Cotroneo, N., Harris, R., Perlmutter, N., Beveridge, T. \& Silverman, J. A. Daptomycin exerts bactericidal activity without lysis of Staphylococcus aureus. Antimicrob. Agents Chemother. 52, 2223-2225 (2008).

18. Pogliano, J., Pogliano, N. \& Silverman, J. A. Daptomycin-mediated reorganization of membrane architecture causes mislocalization of essential cell division proteins. J. Bacteriol. 194, 4494-4504 (2012).

19. Tran, T. T. et al. Daptomycin-resistant Enterococcus faecalis diverts the antibiotic molecule from the division septum and remodels cell membrane phospholipids. mBio 4, e00281-00213 (2013).

20. Hancock, L. E. \& Gilmore, M. S. The capsular polysaccharide of Enterococcus faecalis and its relationship to other polysaccharides in the cell wall. Proc. Natl Acad. Sci. USA 99, 1574-1579 (2002).

21. Laboratories, V. Table of Lectin Properties. http://docs.vectorlabs.com/protocols/ K4-K7.pdf.

22. Beaudoin, T., Zhang, L., Hinz, A. J., Parr, C. J. \& Mah, T. F. The biofilm-specific antibiotic resistance gene $n d v B$ is important for expression of ethanol oxidation genes in Pseudomonas aeruginosa biofilms. J. Bacteriol. 194, 3128-3136 (2012).

23. Whiteley, M. et al. Gene expression in Pseudomonas aeruginosa biofilms. Nature 413, 860-864 (2001)

24. Zhang, L. et al. Identification of genes involved in Pseudomonas aeruginosa biofilm-specific resistance to antibiotics. PLOS ONE. 8, e61625 (2013).

25. Zhang, L., Hinz, A. J., Nadeau, J. P. \& Mah, T. F. Pseudomonas aeruginosa tssC1 links type VI secretion and biofilm-specific antibiotic resistance. J. Bacteriol. 193, 5510-5513 (2011).

26. Zhang, L. \& Mah, T. F. Involvement of a novel efflux system in biofilm-specific resistance to antibiotics. J. Bacteriol. 190, 4447-4452 (2008).

27. Lynch, S. V. et al. Role of the rapA gene in controlling antibiotic resistance of Escherichia coli biofilms. Antimicrob. Agents Chemother. 51, 3650-3658 (2007).

28. Nett, J. et al. Putative role of beta-1,3 glucans in Candida albicans biofilm resistance. Antimicrob. Agents Chemother. 51, 510-520 (2007).

29. Palmer, K. L. et al. Comparative genomics of enterococci: variation in Enterococcus faecalis, clade structure in $E$. faecium, and defining characteristics of E. gallinarum and E. casseliflavus. mBio 3, e00318-00311 (2012).
30. Moormeier, D. E., Bose, J. L., Horswill, A. R. \& Bayles, K. W. Temporal and stochastic control of Staphylococcus aureus biofilm development. mBio 5, e01341-01314 (2014).

31. Banin, E., Vasil, M. L. \& Greenberg, E. P. Iron and Pseudomonas aeruginosa biofilm formation. Proc. Natl Acad. Sci. USA 102, 11076-11081 (2005).

32. O'Toole, G. A., Gibbs, K. A., Hager, P. W., Phibbs, P. V. Jr \& Kolter, R. The global carbon metabolism regulator $\mathrm{Crc}$ is a component of a signal transduction pathway required for biofilm development by Pseudomonas aeruginosa. J. Bacteriol. 182, 425-431 (2000).

33. Petrova, O. E. \& Sauer, K. A novel signaling network essential for regulating Pseudomonas aeruginosa biofilm development. PLoS Pathog. 5, e1000668 (2009).

34. Petrova, O. E., Schurr, J. R., Schurr, M. J. \& Sauer, K. Microcolony formation by the opportunistic pathogen Pseudomonas aeruginosa requires pyruvate and pyruvate fermentation. Mol. Microbiol. 86, 819-835 (2012).

35. Yang, L. et al. Effects of iron on DNA release and biofilm development by Pseudomonas aeruginosa. Microbiology 153, 1318-1328 (2007).

36. Fernandez Guerrero, M. L., Alvarez, B., Manzarbeitia, F. \& Renedo, G. Infective endocarditis at autopsy: a review of pathologic manifestations and clinical correlates. Medicine (Baltimore). 91, 152-164 (2012).

37. Thomas, V. C. et al. A central role for carbon-overflow pathways in the modulation of bacterial cell death. PLoS Pathog. 10, e1004205 (2014).

38. Davies, D. G. et al. The involvement of cell-to-cell signals in the development of a bacterial biofilm. Science 280, 295-298 (1998).

39. Hentzer, M. et al. Alginate overproduction affects Pseudomonas aeruginosa biofilm structure and function. J. Bacteriol. 183, 5395-5401 (2001).

40. Leuck, A. M., Johnson, J. R. \& Dunny, G. M. A widely used in vitro biofilm assay has questionable clinical significance for enterococcal endocarditis. PLOS ONE 9, e107282 (2014).

41. Huycke, M. M., Spiegel, C. A. \& Gilmore, M. S. Bacteremia caused by hemolytic, high-level gentamicin-resistant Enterococcus faecalis. Antimicrob. Agents Chemother. 35, 1626-1634 (1991).

42. Benachour, A. et al. The Enterococcus faecalis sigV protein is an extracytoplasmic function sigma factor contributing to survival following heat, acid, and ethanol treatments. J. Bacteriol. 187, 1022-1035 (2005).

43. Comenge, Y. et al. The CroRS two-component regulatory system is required for intrinsic beta-lactam resistance in Enterococcus faecalis. J. Bacteriol. 185, 7184-7192 (2003).

44. Poole, K. Bacterial stress responses as determinants of antimicrobial resistance. J. Antimicrob. Chemother. 67, 2069-2089 (2012).

45. Suntharalingam, P., Senadheera, M. D., Mair, R. W., Levesque, C. M. \& Cvitkovitch, D. G. The LiaFSR system regulates the cell envelope stress response in Streptococcus mutans. J. Bacteriol. 191, 2973-2984 (2009).

46. Van Laar, T. A., Chen, T., You, T. \& Leung, K. P. Sublethal concentrations of carbapenems alter cell morphology and genomic expression of Klebsiella pneumoniae biofilms. Antimicrob. Agents Chemother. 59, 1707-1717 (2015).

47. Dunny, G. M., Brown, B. L. \& Clewell, D. B. Induced cell aggregation and mating in Streptococcus faecalis: evidence for a bacterial sex pheromone. Proc. Natl Acad. Sci. USA 75, 3479-3483 (1978).

48. Schindelin, J. et al. Fiji: an open-source platform for biological-image analysis. Nat. Methods 9, 676-682 (2012).

49. Erlandsen, S. L., Kristich, C. J., Dunny, G. M. \& Wells, C. L. High-resolution visualization of the microbial glycocalyx with low-voltage scanning electron microscopy: dependence on cationic dyes. The journal of histochemistry and cytochemistry: official journal of the Histochemistry Society 52, 1427-1435 (2004).

50. Barnes, A. M., Ballering, K. S., Leibman, R. S., Wells, C. L. \& Dunny, G. M. Enterococcus faecalis produces abundant extracellular structures containing DNA in the absence of cell lysis during early biofilm formation. mBio. 3, e00193-00112 (2012).

Open Access This article is licensed under a Creative Commons Attribution 4.0 International License, which permits use, sharing, adaptation, distribution and reproduction in any medium or format, as long as you give appropriate credit to the original author(s) and the source, provide a link to the Creative Commons license, and indicate if changes were made. The images or other third party material in this article are included in the article's Creative Commons license, unless indicated otherwise in a credit line to the material. If material is not included in the article's Creative Commons license and your intended use is not permitted by statutory regulation or exceeds the permitted use, you will need to obtain permission directly from the copyright holder. To view a copy of this license, visit http://creativecommons. org/licenses/by/4.0/.

(c) The Author(s) 2017 